\title{
GRAPHICAL QUANTUM ERROR-CORRECTING CODES BASED ON ENTANGLEMENT OF SUBGRAPHS
}

\author{
YUAN LI*,‡, MANTAO XU*,† and QIANG SUN* \\ *Department of Communication Engineering, \\ Shanghai Dianji University, Shanghai 200240, China \\ ${ }^{\dagger}$ School of Computing, University of Eastern Finland, \\ FI-80101 Joensuu, Finland \\ ${ }^{\ddagger} y l i @ s d j u . e d u . c n$
}

Received 6 September 2011

\begin{abstract}
Graphical approach provides a more intuitive and simple way to construct error correction codes. How to obtain generator matrix is the key problem of constructing graphical quantum codes. In this paper, we further generalize the graphical quantum code construction method by entangling its disconnected subgraphs, so that the corresponding generator matrix of quantum nondegenerate codes can be easily obtained. By making use of the method of subgraphs entangling, we also point out its application in adjacency matrix constructions of larger colorable graph and graphical quantum nested codes.
\end{abstract}

Keywords: Quantum error correcting codes; graphical codes; entanglement of subgraphs; nested graphs.

PACS numbers: 03.67.Pp, 03.67.Dd

\section{Introduction}

Quantum error-correcting codes (QECCs) are highly entangled states which are used to prevent damaging effects of decoherence. Since the first quantum code was introduced by Shor in $1995,{ }^{1}$ it was shown that the QECC can be constructed based on classical block codes. This led to an important class of QECC known as Calderbank-Shor-Steane (CSS) codes. $^{2,3}$ Afterwards, a more general quantum code, i.e., quantum stabilizer code (QSC), has been investigated. ${ }^{4}$ Currently, almost all QECCs are constructed with stabilizer whose code space is specified by a tensorproducts Abelian group of Pauli operators.

It is well known that stabilizer state is a multi-qubit pure state, which corresponds to an element in an unique set of eigenvectors with unit eigenvalue of the stabilizer. In coding theory, graph not only is related intimately to the construction of classical error-correcting codes, but also has important applications in QECCs. In Ref. 8, a new and simpler way for constructing QSC called graphical quantum error correction codes (G-QECC) on which more direct intuitions was proposed. 
Generally, a quantum state of a coupled quantum systems may be represented by a graph, where its vertices correspond to physical sub-systems and the edges represent interactions among them. According to the definition, graph state is a set of commuting Pauli operators which can be constructed with corresponding subsystems in a general mathematical graph. ${ }^{5-13}$ Therefore, arbitrary graph does not always correspond to a stabilizer state, and a graph state is the special case of stabilizer state. Since the basic theory on G-QECC was proposed, some researchers have devoted to this topic. ${ }^{9-13}$ For examples, Nest proved that each stabilizer state equates to a graph state under local Clifford operations, ${ }^{12}$ and $\mathrm{Hu}$ and his cooperators investigated the construction of both additive and nonadditive graphical nonbinary quantum codes, and proposed some graph codes. ${ }^{13}$

Generally, to construct graphical quantum code, a finite abelian group as stabilizer, whose order is the dimension of the Hilbert space, is used to describe a quantum system. Therefore, how to easily obtain the generators of the stabilizer corresponding to one graph state, is the key problem of constructing for graphical quantum codes. This motivates us to seek the more general construction method of graphical quantum codes. In this paper, we present a new construction method of graphical quantum code by entangling its several disconnected subgraphs, so that the obtained higher-dimension graphical quantum code corresponds to a graph state. Furthermore, Graph states associated with colorable graph, have some useful properties. $^{8,13-18}$ By making use of the present method, we shall also consider its application in the adjacent matrix construction of large colorable graph. Furthermore, how to gain the adjacency matrix of graphical quantum nested codes is a difficult problem. With the one-to-one entanglement method, the constructed colorable nested graph via its subgraphs can possess of optimal coloring according to the graph coloring problem.

The paper is structured as follows. In Sec. 2, we introduce some preliminaries which are necessary for G-QECC. In Sec. 3, we investigate the construction approach with its disconnected subgraphs to obtain graphical quantum codes, and its application into the adjacency matrix constructions for larger colorable graph and nested graph. Furthermore, we also investigate the bounds that good G-QECCs should satisfy in Sec. 4. Finally, conclusions are drawn in Sec. 5.

\section{Preliminaries for Graphical Quantum Codes Construction}

A graph is a collection of vertices which are connected by edges. Formally, an undirected and finite graph is a pair

$$
G=(V, E),
$$

where the elements of $V$ are called vertices, and the elements of $E$ edges. Denote $N=|V|$ the vertex number of graph $G$. In an $N$-qubit quantum network graph, a Pauli group with tensor products may be generated

$$
\mathcal{P}^{V}=\left\{i^{\lambda}\left(\tau_{1} \otimes \cdots \otimes \tau_{N}\right) \mid 0 \leq \lambda \leq 3, \tau_{j} \in \mathcal{P}, 1 \leq j \leq N\right\},
$$


where $i=\sqrt{-1}$ and $\mathcal{P}=\left\{\sigma_{0}, \sigma_{x}, \sigma_{z}, \sigma_{y}\right\}$ is a set composed of four basic Pauli matrices. Any pair of elements in $\mathcal{P}^{V}$ either commutes or anti-commutes, and graph $G$ corresponds to a $N$-qubit graph system in space $\mathcal{P}^{V}$. Stabilizer code is known as a class of quantum code which is given by the most widely-used structure. Let $S$ be an abelian subgroup of $\mathcal{P}_{N}$, namely, any two vectors in $S$ commute. Acting each element in $S$ onto joint eigenspace associated to state in quantum codes space, if the eigenvalue is $1, S$ is called the stabilizer of the quantum code and its element is called generator.

The adjacency matrix $\Gamma$ of a simple graph, is to describe the connection relation between all vertices. It is an $N \times N$ symmetric matrix for the system corresponding to a nondegenerate code and consisting of $N$ qubits with elements

$$
\Gamma_{a b}= \begin{cases}1 & \text { if }(a, b) \in E \\ 0 & \text { otherwise. }\end{cases}
$$

Because of the entanglement between $N$ vertices, each vertex $a$ in graph $G$ corresponds uniquely to an $N$-qubit vector. At each qubit in a vertex vector, we use the following encoding of the Pauli matrices in $\mathcal{P}$ as pairs of bits with two types (i.e., $X$-type and $Z$-type)

$$
\sigma_{0} \rightarrow(0 \mid 0), \quad \sigma_{x} \rightarrow(1 \mid 0), \quad \sigma_{y} \rightarrow(1 \mid 1), \quad \sigma_{z} \rightarrow(0 \mid 1) .
$$

Namely, any one $N$-fold vertex vector $\mathcal{M}_{a} \in \mathcal{P}^{V}, a \in V$, corresponds to an $2 N$-dimensional binary vector

$$
\mathbf{a}=\left(a_{1}^{x} a_{2}^{x} \cdots a_{N}^{x} \mid a_{1}^{z} a_{2}^{z} \cdots a_{N}^{z}\right) \in Z_{2}^{2 N},
$$

where $a_{i}^{x}, a_{i}^{z} \in\{0,1\}$ for $i=1, \ldots, N$.

An arbitrary mathematical graph does not always corresponds to a stabilizer state, unless its vertex vectors satisfy the commutating condition. Therefore, graph states are special cases of stabilizer states, which are certain pure multi-particle entangled states associated with graphs. ${ }^{12}$ If any vertex in graph $G$ corresponds to a generator $\mathcal{M}_{a}$ of stabilizer $S$, then graph state $|G\rangle$ equates to $S$. Therefore, the vertex vector $\mathcal{M}_{a}$ in space $\mathcal{P}^{V}$ may be expressed in graph language as

$$
\mathcal{M}_{a}=X^{a} \bigotimes_{b \in N(a)} Z^{b}
$$

which is associated with its adjacent vertices, where $N_{a}:=\{b \in V \mid a, b \in E\}$ is the adjacent vertex collection for a given vertex $a \in V$ in graph $G=(V, E)$. By applying on vertex $a$ in $G$ respectively, $X^{a}, Y^{a}, Z^{a}$ denote Pauli matrices $\sigma_{x}, \sigma_{y}$, $\sigma_{z}$ in (4), where

$$
X^{a}=\sum_{y \in \mathbb{Z}_{2}^{N}}|y+a\rangle\left\langle y\left|, \quad Z^{a}=\sum_{y \in \mathbb{Z}_{2}^{N}} \omega^{a y}\right| y\right\rangle\langle y|, \quad \omega=e^{i \pi}
$$

and $Y^{a}=i X^{a} Z^{a}$ over $\mathbb{Z}_{2}^{N}$. Therefore, the generator matrix of a graph state can be expressed as binary standard form

$$
\mathcal{G}=(X \mid Z)=(I \mid \Gamma),
$$


where $\Gamma$ is the adjacency matrix of the graph $G$. For simplicity, it also can be directly described over $G F(4)=\left\{0,1, \omega, \omega^{2}\right\}$ as

$$
C=\omega I+\Gamma
$$

for mapping: $\omega \rightarrow \sigma_{x}$. Let $\mathcal{M}_{a}$ and $\mathcal{M}_{b}$ be two commuting vertex vectors in $S$ corresponding to binary vectors $\mathbf{a}$ in $(5)$ and $\mathbf{b}=\left(b_{1}^{x} b_{2}^{x} \cdots b_{N}^{x} \mid b_{1}^{z} b_{2}^{z} \cdots a_{N}^{z}\right)$ respectively. According to the definition of stabilizer, their symplectic product equates zero, i.e.,

$$
\mathbf{a} \cdot \mathbf{b}=\sum_{i=1}^{N}\left(a_{i}^{x} \cdot b_{i}^{z}+a_{i}^{z} \cdot b_{i}^{x}\right)=0 .
$$

Graphical quantum codes $\mathcal{C}[[|V|, k, d]]$ encodes vectors from $2^{k}$-dimensional complex vector space to $2|V|$-dimensional Hilbert space $\mathbb{H}_{|V|}=\left(\mathbb{C}^{2}\right)^{\otimes|V|}$, where $\mathbb{C}^{2}$ be an 2-dimension complex vector space. A coding clique $\mathcal{C}(K, d)$ of a given graph $G=(V, E)$ encodes $|V|-k$ vertices for $|K|=k$ into $|V|$-qubit graph $G$, where $K$ is a collection of $k$ different vertex vectors in the graph. Obviously, it is that $\mathbf{0} \in \mathcal{C}(K, d)$ and each vector $s \in S$ is orthogonal to any vectors $c \in \mathcal{C}(K, d)$. Furthermore, the coding clique $\mathcal{C}(K, d)$ is close and linear. The parameters of G-QECC should also satisfy some quantum bound, such as Singleton bound ${ }^{19}$

$$
|V|-|K| \geq d-1 \text {. }
$$

So, one obtains a graph clique code $\mathcal{C}(K, d)$ by selecting $N-k$ vertex vectors from the generators of graph state $|G\rangle$ with bound.

\section{Graphical Quantum Codes Construction by Entangling Subgraphs}

Clearly, one may see that the key problem of constructing G-QECC is how to obtain its generator matrix. As the following, we will present a method of obtaining a new graph by entangling several disconnected subgraphs.

\subsection{Multi-vertex quantum graph code construction method via entanglement}

A graph $G_{0}=\left(V_{0}, E_{0}\right)$ is called a subgraph of $G=(V, E)$, if $V_{0} \subset V$ and $E_{0} \subset E$. There is no any $\{a, b\}$-path to connect two subgraphs $G_{1}=\left(V_{1}, E_{1}\right)$ and $G_{2}\left(V_{2}, E_{2}\right)$ of graph $G$ for $a \in V_{1}, b \in V_{2}$, then the two subgraphs are called disconnected. As the following, we shall introduce an approach of obtaining a graph by entangling its several disconnected subgraphs.

Without loss of generality, we consider how to entangle two disconnected subgraphs $G_{1}=\left(V_{1}, E_{1}\right)$ and $G_{2}=\left(V_{2}, E_{2}\right)$, so that a new graph $G=(V, E)$ can be gained, where $G_{1}$ and $G_{2}$ correspond to coding cliques $\mathcal{C}_{G_{1}}\left(K_{1}, d_{1}\right) \cap \mathcal{C}_{G_{2}}\left(K_{2}, d_{2}\right)$, respectively. Define an auxiliary graph

$$
G^{\prime}=G_{1} \oplus G_{2}
$$


as a graph formed by the two disconnected subgraphs which share no edge. Assume $\Gamma_{i}$ are the adjacent matrices of graph $G_{i}$ for $i=1,2$ respectively, the binary standard form of generator matrix of graph $G^{\prime}$ is

$$
\mathcal{G}^{\prime}=(I \mid \Gamma)=\left(\begin{array}{cc|cc}
I_{1} & \mathbf{0} & \Gamma_{1} & \mathbf{0} \\
\mathbf{0} & I_{2} & \mathbf{0} & \Gamma_{2}
\end{array}\right),
$$

where $I_{i}$ is an $N_{i} \times N_{i}$ unitary matrix for $N_{i}=\left|V_{i}\right|$. Extend the matrix (13) to more general form

$$
\mathcal{G}=(I \mid \Gamma)=\left(\begin{array}{cc|cc}
I_{1} & \mathbf{0} & \Gamma_{1} & \Gamma_{12} \\
\mathbf{0} & I_{2} & \Gamma_{12}^{T} & \Gamma_{2}
\end{array}\right),
$$

where $\Gamma_{12}$ is called $N_{1} \times N_{2}$ entanglement relation matrix and $\Gamma_{12}^{T}$ is its reverse matrix. Corresponding to form (14), there must be a graph $G=(V, E)$ with $V=$ $V_{1} \cup V_{2}$ may be obtained.

Because an arbitrary graph does not correspond to a graph state, another problem which must be considered is that the obtained graph $G$ whether can be used to construct quantum codes. Namely, any two vertex vectors in the obtained graph should commute so that the symplectic condition (10) is satisfied.

Theorem 1. Assume a graph $G=(V, E)$ with the standard form in Eq. (14) is entangled by two disconnected subgraphs $G_{1}=\left(V_{1}, E_{1}\right)$ and $G_{2}=\left(V_{2}, E_{2}\right)$ with respect to entanglement relation matrix $\Gamma_{12}$. If the subgraphs correspond to two graph states, then the entangled graph also corresponds to a graph state. Furthermore, the distance is no less than each distance of the subgraph codes when connection matrix $\Gamma_{12}$ is a full-rank matrix.

Proof. To illuminate the obtained graph $G$ corresponds to a stabilizer state, we only prove its any two vertex vectors in generator matrix $C$ commute. Assume vector $\boldsymbol{\alpha}_{s}=\left(a_{s 1}^{x}, \ldots, a_{s N}^{x} \mid a_{s 1}^{z}, \ldots, a_{s N}^{z}\right), 1 \leq s \leq N=|V|$, is an any vector in the obtained graph $G$, and $N_{1}=\left|V_{1}\right|, N_{2}=\left|V_{2}\right|$. Two cases on a pair of vectors should be considered as following.

Case 1. Consider two vectors $\boldsymbol{\alpha}_{i}$ and $\boldsymbol{\alpha}_{j}$, for $1 \leq i, j \leq N_{1}$ or $N_{1}+1 \leq i, j \leq$ $N_{1}+N_{2}$. Without loss of generality, let the two vectors be among first $N_{1}$ rows of generator matrix $C$. Then, their symplectic product should be

$$
\boldsymbol{\alpha}_{i} \cdot \boldsymbol{\alpha}_{j}=\sum_{h=1}^{N_{1}+N_{2}} a_{i h}^{x} a_{j h}^{z}+\sum_{h=1}^{N_{1}+N_{2}} a_{i h}^{z} a_{j h}^{x} .
$$

Note that $G_{1}$ corresponds to a graph state, i.e., $a_{i l}^{x}=a_{j l}^{x}=0$ for $N_{1}+1 \leq l \leq$ $N_{1}+N_{2}$. One may hence get

$$
\boldsymbol{\alpha}_{i} \cdot \boldsymbol{\alpha}_{j}=\sum_{h=1}^{N_{1}} a_{i h}^{x} a_{j h}^{z}+\sum_{h=1}^{N_{1}} a_{i h}^{z} a_{j h}^{x}=0 .
$$


Similarly, when $N_{1}+1 \leq i, j \leq N_{1}+N_{2}$, because $G_{2}$ also corresponds to a graph state, it can be induced that their symplectic product equates zero.

Case 2. Assume two vectors $\boldsymbol{\alpha}_{i}$ and $\boldsymbol{\alpha}_{j}$ belong respectively to the first $N_{1}$ vectors and later $N_{2}$ vectors in the generator matrix $C$, i.e., $1 \leq i \leq N_{1}$ and $N_{1}+1 \leq j \leq$ $N_{1}+N_{2}$. Then, their symplectic product should be

$$
\begin{aligned}
\boldsymbol{\alpha}_{i} \cdot \boldsymbol{\alpha}_{j} & =\sum_{h_{1}=1}^{N_{1}+N_{2}} a_{i h_{1}}^{x} a_{j h_{1}}^{z}+\sum_{h_{2}=1}^{N_{1}+N_{2}} a_{j h_{2}}^{x} a_{i h_{2}}^{z} \\
& =\sum_{h_{1}=1}^{N_{1}} a_{i h_{1}}^{x} a_{j h_{1}}^{z}+\sum_{h_{2}=N_{1}+1}^{N_{1}+N_{2}} a_{j h_{2}}^{x} a_{i h_{2}}^{z} \\
& =a_{i i}^{x} a_{j i}^{z}+a_{j j}^{x} a_{i j}^{z} \\
& =a_{j i}^{z}+a_{i j}^{z} .
\end{aligned}
$$

Because $a_{i j}^{z}$ and $a_{j i}^{z}$ belong to entanglement relation matrices $\Gamma_{12}$ and $\Gamma_{12}^{T}$ respectively, one may get $a_{j i}^{z}=a_{i j}^{z}$. This implies that their symplectic product equates zero with module 2 , namely, two vectors commute.

In summary, any two vectors in the obtained graph always commute, so each one corresponds to a generator of graph state. Furthermore, one may easily get that the distance of formed new graphical quantum code is no less than any one of the distance of subgraphs. Specially, it is larger than single distance when the entanglement relation matrix is square and full-rank.

From the description we may see that, a new graphical quantum code by entangling its subgraphs hence can be obtained, by making rows collection of the corresponding generator matrix as the generators set $\left\{\mathcal{M}_{a_{i}}, 1 \leq i \leq|V|, a_{i} \in V\right\}$. Generally, in two cases on entanglement relation matrix $\Gamma_{12}$, the generator matrix $C$ in (9) of graph $G$ can be gained by entangling its two subgraphs.

Case 1. If $N_{1}=N_{2}$, we take an $N / 2$ by $N / 2$ full-rank square matrix $\Gamma_{12}$ to generate the new graph.

Case 2. If $N_{1} \neq N_{2}$ such that $m N_{1}<N_{2}<(m+1) N_{1}$ for some integer $m$, we take the $N_{1}$ by $N_{2}$ entanglement relation matrix $\Gamma_{12}$ as

$$
\Gamma_{12}=\left(\Gamma_{A_{12}^{(1)}}, \ldots, \Gamma_{A_{12}^{(m)}}, \Gamma_{B_{12}}\right),
$$

where at least one of $\Gamma_{A_{12}^{(i)}}, 1 \leq i \leq m$, is an $N_{1}$ by $N_{1}$ full-rank square matrix, and $\Gamma_{B_{12}}$ is an $N_{1}$ by $N_{2}-m N_{1}$ matrix with rank $r=\left\{\min N_{1}, N_{2}-m N_{1}\right\}$.

The other one left problem is how to design the entanglement relation matrix $\Gamma_{12}$ to entangle its subgraphs, so that the obtained graph $G$ corresponds to a quantum graphical code with parameters $\left[\left[N=N_{1}+N_{2}, k, d \geq \min \left\{d_{1}, d_{2}\right\}\right]\right]$. Considering 
the Theorem and boundary conditions, the entanglement relation matrix $\Gamma_{12}$ should be constructed with following principles.

(1) To get a more steadier state and increase the correction capability, matrix $\Gamma_{12}$ should be full rank so that the maximal clique of entangled graph may be obtained. Namely, a maximally entangled pair of two-spin system can be obtained when the minimal kernel dimension of entanglement relation matrix $\Gamma_{12}$ may be rank $r=\min \left\{N_{1}, N_{2}\right\}$.

(2) For simple encoding, the entanglement relation matrix $\Gamma_{12}$ should be a lowdensity matrix, i.e., it can be divided into several block matrices, and one sub-matrix may be transferred to lower triangular matrix.

Define a cycle (or circuit) of graph, which is a path that starts and ends at the same vertex.

(3) Generally, a long cycle should be arranged in such construction, that no smaller cycles are contained in subgraphs, including the matrix $G_{12}$ in entanglement relation graph. This induces the increasing capability of obtained graphical quantum codes.

(4) Denote $w$ the weight of vector in matrix $\Gamma_{12}$. Then, $w$ will be no more than $w_{0}=\min \left\{k_{1}, k_{2}\right\}-1$.

Furthermore, a graph $G_{12}=\left(V_{12}, E_{12}\right)$ generated from $N \times N=\left(N_{1}+N_{2}\right) \times$ $\left(N_{1}+N_{2}\right)$ adjacency matrix

$$
\Gamma_{G_{12}}=\left(\begin{array}{ll}
\mathbf{0} & \Gamma_{12} \\
\Gamma_{12}^{T} & \mathbf{0}
\end{array}\right),
$$

clearly, is also a subgraph of $G$, and it has following edges relation

$$
E=E_{1} \cup E_{2} \cup E_{12}
$$

Therefore, assume graphs $G^{\prime}$ with (13) and $G_{12}$ with (19) generate two coding cliques $\mathcal{C}_{G^{\prime}}\left(K^{\prime}, d^{\prime}\right)$ and $\mathcal{C}_{G_{12}}\left(K_{12}, d_{12}\right)$. Then, coding clique $\mathcal{C}_{G}(K, d)$ generated from the graph $G$ may be expressed as

$$
\mathcal{C}_{G}(K, d)=\mathcal{C}_{G^{\prime}}\left(K_{1}, d_{1}\right) \cap \mathcal{C}_{G_{12}}\left(K_{12}, d_{12}\right) .
$$

More general, it easily can be generalized for entangling several subgraphs to obtain a new network graph. Note that the Schmidt rank of a graph state is closely related to error correction capability of a corresponding graph code. According to Ref. 8, to encode $k$-dimension subset $K \subset V$ as an input on vertices, we take corresponding output on vertices $M=V-K$ in $G$ to detect the error configuration $E$. Therefore, any vertex number $|E|-1$ of errors can be detected and one half of which be corrected. Physically, by applying controlled-phase gate on the initialized graph state in terms of adjacency matrix $\Gamma$ in (14), the preparation of the graph state correspond to quantum code $\mathcal{C}_{G}(K, d)$ can be accomplished. 
Coloring is an assignment of colors to the vertices of a graph, such that no two adjacent vertices have the same color. A graph $G=(V, E)$ is generally called $k$-colorable if one can divide the vertices into $k$ groups

$$
V_{1}, V_{2}, \ldots, V_{k} \subset V
$$

and assign to the vertices of each group a color, such that neighboring vertices have different colors. ${ }^{18}$ Specially, two-colorable graph (also called bipartite graph) is an instance with $k=2$, of which the vertices set is partitioned into two disjoint sets, and no two vertices within the same set are adjacent. Graph states associated with colorable graph, have some useful properties, such as, the form and color of the vertices can indicate the different measurement directions or the respective vertex can correspond to an input and output qubit. For example, two-colorable graph states are equivalent to CSS states. ${ }^{14}$ The smallest integer $k$ such that $G$ has an $k$-coloring is called the chromatic number of $G$ and is denoted by $\mathcal{X}(G)$. Graph coloring problem is to find the fewest possible colors in graph.

To obtain a more multi-vertex colorable graph and containing colors as few as possible, based on the present entanglement method, the relation between the colors of entangled graph $G$ and its two subgraphs should be considered. It requests that each vertex in subgraph of less colors should entangle a vertex in every divided subsets except for its same-color subset.

Proposition 1. Assume $G_{1}$ and $G_{2}$ are two disconnected graphs of $k_{1}$ and $k_{2}$ colors, respectively, and correspond to two graph states.

Then, the colors of graph $G$ by entangling its subgraphs $G_{1}$ and $G_{2}$ will be no more than $k_{1}+k_{2}$. Specially, when matrix $\Gamma_{12}$ describes the one-to-one mapping between different colorable vertices in respective subgraphs, the coloring number of graph $G$ should be $k=\max \left\{k_{1}, k_{2}\right\}$.

Therefore, by combining several colorable subgraphical quantum states, a larger colorable graph state can be obtained.

Example 1. Consider an example for case of $N_{1}=N_{2}$. Let

$$
C_{6}^{\prime}=\Gamma_{6}^{\prime}+\omega I=\left(\begin{array}{cccccc}
0 & 1 & 0 & 1 & 0 & 1 \\
1 & 0 & 1 & 0 & 1 & 0 \\
0 & 1 & 0 & 1 & 0 & 1 \\
1 & 0 & 1 & 0 & 1 & 0 \\
0 & 1 & 0 & 1 & 0 & 1 \\
1 & 0 & 1 & 0 & 1 & 0
\end{array}\right)
$$


and

$$
C_{6}^{\prime \prime}=\Gamma_{6}^{\prime \prime}+\omega I=\left(\begin{array}{cccccc}
0 & 1 & 0 & 0 & 0 & 1 \\
1 & 0 & 1 & 0 & 0 & 0 \\
0 & 1 & 0 & 1 & 0 & 0 \\
0 & 0 & 1 & 0 & 1 & 0 \\
0 & 0 & 0 & 1 & 0 & 1 \\
1 & 0 & 0 & 0 & 1 & 0
\end{array}\right)
$$

be two generator matrices of two-colorable subgraphs $G^{\prime}$ and $G^{\prime \prime}$, which based on adjacent matrices $\Gamma_{6}^{\prime}$ and $\Gamma_{6}^{\prime \prime}$ corresponding to two graph states, respectively. Take a simple one-to-one entanglement relation matrix as

$$
\Gamma_{12}=\left(\begin{array}{cccccc}
0 & 1 & 0 & 0 & 0 & 0 \\
0 & 0 & 1 & 0 & 0 & 0 \\
0 & 0 & 0 & 1 & 0 & 0 \\
0 & 0 & 0 & 0 & 1 & 0 \\
0 & 0 & 0 & 0 & 0 & 1 \\
1 & 0 & 0 & 0 & 0 & 0
\end{array}\right)
$$

Then, based on two colorable subgraphs, the obtained graph corresponding to code [[12, 0,4]] can be seen Fig. 1 .

Example 2. Consider case of $N_{1} \neq N_{2}$. Assume $G_{1}$ and $G_{2}$ correspond to two three-colorable graph states, and their generator matrices are

$$
C_{1}=\Gamma_{1}+\omega I=\left(\begin{array}{ccc}
\omega & 1 & 1 \\
1 & \omega & 1 \\
1 & 1 & \omega
\end{array}\right)
$$

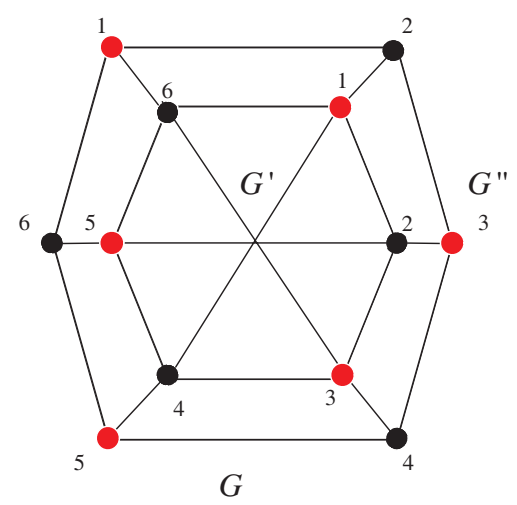

Fig. 1. Two-colorable graph quantum nested code $[[12,0,4]]$ based on colorable subgraphs. 
and

$$
C_{2}=\Gamma_{2}+\omega I=\left(\begin{array}{ccccccc}
\omega & 1 & 0 & 1 & 1 & 0 & 1 \\
1 & \omega & 1 & 0 & 1 & 1 & 0 \\
0 & 1 & \omega & 1 & 0 & 1 & 1 \\
1 & 0 & 1 & \omega & 1 & 0 & 1 \\
1 & 1 & 0 & 1 & \omega & 1 & 0 \\
0 & 1 & 1 & 0 & 1 & \omega & 1 \\
1 & 0 & 1 & 1 & 0 & 1 & \omega
\end{array}\right)
$$

Based on the two matrices, $G^{\prime}$ in (12) can be easily obtained. Next, we take the entanglement relation matrix as

$$
\Gamma_{B_{12}}^{T}=\left(\begin{array}{ccccccc}
0 & 1 & 0 & 1 & 1 & 0 & 1 \\
0 & 0 & 1 & 0 & 0 & 1 & 0 \\
1 & 0 & 0 & 0 & 0 & 0 & 1
\end{array}\right)
$$

So, we can obtain the generator matrix of multi-vertex quantum code $[[12,0,4]]$ corresponding to three-colorable graph $G$ in Fig. 2.

Lemma 1. Generally, define direct sum of $N$ full-rank matrices as

$$
A=A_{1} \oplus A_{2} \oplus \cdots \oplus A_{N}=\left(\begin{array}{cccc}
A_{1} & 0 & \cdots & 0 \\
0 & A_{1} & \cdots & 0 \\
\vdots & \vdots & \ddots & \vdots \\
0 & 0 & \cdots & A_{N}
\end{array}\right)
$$

Then, it has some properties as following:

(i) $\operatorname{rank}(A)=\sum_{i=1}^{N} \operatorname{rank}\left(A_{i}\right)$;

(ii) If $A_{1}, \ldots, A_{N}$ are all orthogonal matrices, their $\operatorname{direct}$ sum $A$ is also orthogonal.

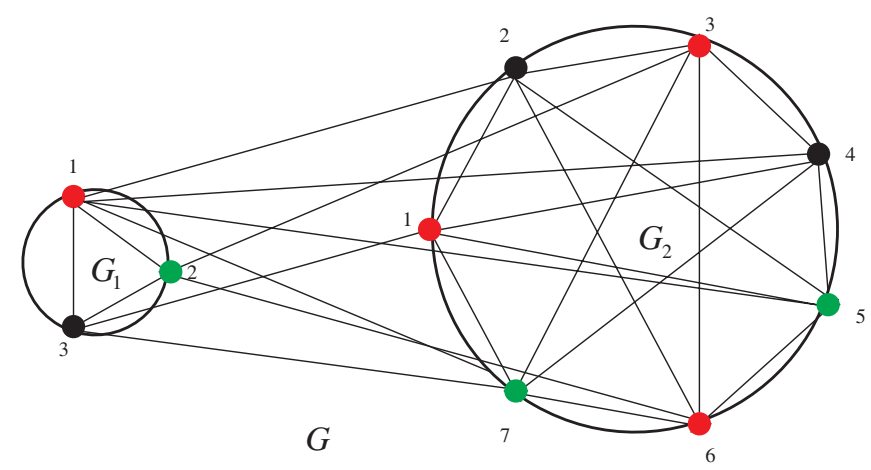

Fig. 2. Three-colorable graph $G$ with length 12 , correcting one error. 
Take $(N-1) N / 2$ full-rank entanglement relation matrices to replace the zero matrices in (29), if $A_{i}, 1 \leq i \leq N$, all correspond to graph states, then matrix $A$ may be a generator matrix of graphical quantum code of larger distance.

\subsection{The application of present construction method to graphical quantum nested code}

In practice, the proposed approach in previous section provides a more general way to obtain graphical quantum codes. It has some valuable applications quantum codes construction theory, such as the construction of quantum nested graph codes. As the following, we apply the construction technique based on entanglement to obtain the adjacency matrix of nested colorable-graph quantum codes.

A nested graph $G=(V, E)$, can be described as an " $n_{l}$-vertices of $\ldots$ graph of $n_{2}$-vertices graph of $n_{1}$-vertices graph", denoted by

$$
G_{n_{l}}\left[\cdots\left[G_{n_{2}}\left[G_{n_{1}}\right]\right] \cdots\right] .
$$

According to the definition, one may see that the number of vertices in the graph (30) is $N=n_{1} n_{2} \cdots n_{l}$, and the vertices of obtained graph $G$ is formed by $N / n_{1}$ disjoint colorable vertex subsets $V_{1}, V_{2}, \ldots, V_{N / n_{1}}$ with same size $n_{1}$, where $V_{i}$ for $1 \leq i \leq l$ is the vertex set of graph $G_{n_{1}}$. So, it results in

$$
V=V_{1} \cup V_{2} \cup \cdots \cup V_{N / n_{1}},
$$

and its every subgraph is same to graph $G_{n_{1}}$. Let $E_{i j}$ be the set of edges $\{u, v\}$ where $u$ and $v$ respectively belong to two sets $V_{i}$ and $V_{j}$. One may get the number $\left|E_{i j}\right|$ is $n_{1}$, namely, every vertex in $V_{i}$ connects to one vertex in $V_{j}$ and vice versa. Therefore, the obtained entangling graph state has at most $V_{N / n_{1}}$ colorable subgraphs. Graph coloring problem is to find a coloring with as few colors as possible. Therefore, one should take a simple connection relation to entangle the subgraphs, so that the obtained entangled graph has fewer colors.

According to its characters, a nested graph actually is formed by connecting $N / n_{1}$ subgraphs which are complete same to $G_{n_{1}}$. Therefore, for generating the adjacency matrix $\Gamma$ of graph $G$, the key problem is how to connect the subgraphs with given adjacency matrix $\Gamma_{1}$ of $G_{n_{1}}$. As the following, we introduce a family of entanglement relation matrices. Let a finite group

$$
\mathcal{T}_{L}=\left\{P_{L}^{0}=I, P_{L}, \ldots, P_{L}^{L-1}\right\},
$$

where $P_{L}=\left(p_{i j}\right)$ is a circulant permutation matrix defined as

$$
p_{i j}= \begin{cases}1 & \text { if } i=(j+1) \bmod L \\ 0 & \text { otherwise }\end{cases}
$$

If $G_{n_{1}}$ is a colorable subgraph, to optimize coloring we take $\Gamma_{12} \in \mathcal{T}_{n_{1}}$ as the one-toone entanglement relation matrix, so that subgraph $G_{n_{2}}\left[G_{n_{1}}\right]$ can be obtained. More 
general, if $\Gamma_{n_{i-1}}$ is the adjacency matrix of nested subgraph $G_{n_{i-1}}\left[\cdots\left[G_{n_{2}}\left[G_{n_{1}}\right]\right] \cdots\right]$ for $1<i \leq l$, then the adjacency matrix of graph $G_{n_{i}}\left[\cdots\left[G_{n_{2}}\left[G_{n_{1}}\right]\right] \cdots\right]$ is

$$
\Gamma_{n_{i}}=\left(\begin{array}{cccc}
\Gamma_{n_{i-1}} & \Gamma_{i_{12}} & \ldots & \Gamma_{i_{1\left(n_{i}-1\right)}} \\
\Gamma_{i_{12}}^{T} & \Gamma_{n_{i-1}} & \ldots & \Gamma_{i_{2\left(n_{i}-1\right)}} \\
\vdots & \vdots & \vdots & \vdots \\
\Gamma_{i_{1\left(n_{i}-2\right)}}^{T} & \Gamma_{i_{2\left(n_{i}-2\right)}}^{T} & \ldots & \Gamma_{i_{\left(n_{i}-1\right)\left(n_{i}-1\right)}} \\
\Gamma_{i_{1\left(n_{i}-1\right)}}^{T} & \Gamma_{i_{2\left(n_{i}-1\right)}}^{T} & \cdots & \Gamma_{n_{i-1}}
\end{array}\right),
$$

where $\Gamma_{i_{s t}}=P^{m_{s t}} \in \mathcal{T}_{n_{i-1}}$ for $1 \leq s, t \leq n_{i}-1,0 \leq m_{s t} \leq n_{i-1}$ are entanglement relation matrices. If there is no intercross between any pair of colorable notes in different subgraphs, then the exponent $m_{s t}$ should satisfy

$$
\sum_{s, t=1}^{n_{i}-1} m_{s t} \bmod n_{i}=0, n_{i}>2,
$$

and $P_{s t}^{m}$ is any element in $\mathcal{T}_{n_{i-1}}$ when $n_{i}=2$.

Furthermore, consider the error correction capability of the generated quantum code. According to the described results, if graph $G_{n_{1}}$ corresponds to a graph state, then the obtained nested graph $G$ still do in terms of the theorem. So, one can construct a graphical quantum code with parameters $[[|V|, k, d]]$. Note that the entanglement relation matrices generated from set of circulant permutation matrices in (33), i.e., they are all full-rank. Note that every sub-block matrices is square. Therefore, it is obviously that the distance of obtained graphical quantum nested code $[[|V|, k, d]]$ is larger than that of its any single subgraphs $G_{n_{s}}\left[\cdots\left[G_{n_{1}}\right] \cdots\right]$ for $1 \leq s \leq l-1$.

Example 3. Consider the construction for nested colorable graph $G_{3}\left[G_{4}\right]$, where the generator matrices of two-colorable subgraph $G_{4}$ and graph $G_{3}$ over GF(4) are

$$
C_{4}=\Gamma_{4}+\omega I_{4}=\left(\begin{array}{cccc}
\omega & 1 & 0 & 1 \\
1 & \omega & 1 & 0 \\
0 & 1 & \omega & 1 \\
1 & 0 & 1 & \omega
\end{array}\right)
$$

and

$$
C_{3}=\Gamma_{3}+\omega I_{3}=\left(\begin{array}{ccc}
\omega & 1 & 1 \\
1 & \omega & 1 \\
1 & 1 & \omega
\end{array}\right) .
$$

Furthermore, the relation matrices are the elements of $\mathcal{T}_{4}$. So, one may get that the 
adjacency matrix $\Gamma_{12}$ of nested graph $G_{3}\left[G_{4}\right]$ is

$$
\Gamma=\left(\begin{array}{ccc}
\Gamma_{4} & P_{4} & P_{4}^{2} \\
\left(P_{4}\right)^{T} & \Gamma_{4} & P_{4} \\
\left(P_{4}^{2}\right)^{T} & \left(P_{4}\right)^{T} & \Gamma_{4}
\end{array}\right)
$$

Matrix $\Gamma$ of graph $G$ with no intercross between any pair of subgraphs may also be taken as

$$
\left(\begin{array}{ccc}
\Gamma_{4} & P_{4} & P_{4} \\
\left(P_{4}\right)^{T} & \Gamma_{4} & P_{4}^{2} \\
\left(P_{4}^{2}\right)^{T} & \left(P_{4}^{2}\right)^{T} & \Gamma_{4}
\end{array}\right), \quad\left(\begin{array}{ccc}
\Gamma_{4} & I & P_{4}^{3} \\
I & \Gamma_{4} & P_{4} \\
\left(P_{4}^{3}\right)^{T} & \left(P_{4}\right)^{T} & \Gamma_{4}
\end{array}\right)
$$

and so on. Finally, one may obtain the generator matrix $C=\Gamma+\omega I_{12}$. Correspondingly, one may gain a quantum nested graph code $[[12,0,6]]$ using to graph $G_{3}\left[G_{4}\right]$ based on the entanglement relation matrix (38), which may be seen Fig. 3.

In terms of relevant adjacent matrices, based on their colorable subgraphs, some other general nested graph states with higher level may be obtained, such as colorable graph in Fig. 4.

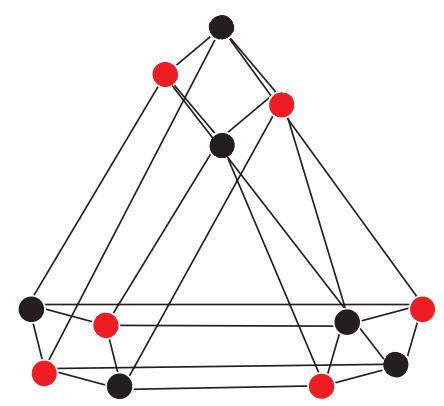

Fig. 3. Two-colorable nested graph $G_{3}\left[G_{4}\right]$ of quantum code $[[12,0,6]]$ based on its colorable subgraphs.

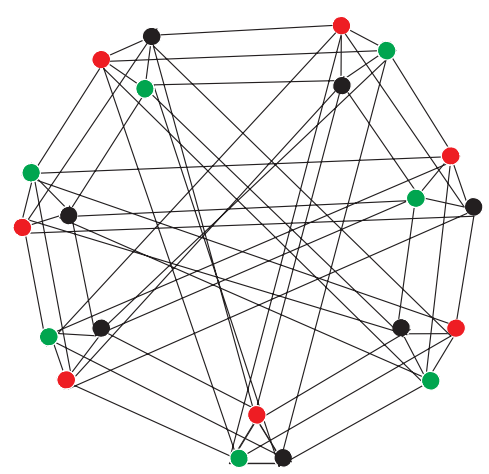

Fig. 4. Three-colorable nested graph $G_{7}\left[G_{3}\right]$ of quantum code $[[21,0,9]]$. 


\section{Bounds of Good Graphical Quantum Codes}

Now, we consider the bound of good G-QECCs. According to our present approach, one may get that the parameters $\delta=d / N$ and $R=k / N$ can be adjusted by choose proper connection matrices and subgraphs. As the following, we consider the parameters should satisfy the bounds to make the obtained graphs correspond to the good G-QECCs.

A probabilistic graph is a graph equipped with a probability distribution on its vertices. ${ }^{20}$ Let $(G, p)$ be a probabilistic graph, and $X$ be any random variable over the vertex set $V(G)$ of $G$ with distribution $p$. Graph entropy $H(G, p)$ is an information theoretic functional of a graph $G$ with a probability distribution $p$ on its vertex set. Let $F$ and $G$ be two graphs on the same vertex set $V$ with possibly intersecting edge sets. We recall that the mutual information $I(X \wedge Y)$ of the random variables $X$ and $Y$ equals $H(X)+H(Y)-H(X, Y)$, where $H(X, Y)$ is the entropy of the random variable $(X, Y)$. Then, the graph entropy is formally defined as

$$
H(G, p)=\min _{X \in Y \in S(G), P_{X}=p} I(X \wedge Y),
$$

where $S(G)$ denotes the family of the stable sets of vertices in $G$.

Graph entropy $\mathrm{H}(\mathrm{G} ; \mathrm{p})$ is an information theoretic functional of a graph $\mathrm{G}$ with a probability distribution $\mathrm{p}$ on its vertex set. For an arbitrary integer $\mathrm{m}$, we can select the perimeter $\mathrm{k}$ such that.

We now analyze the asymptotic good behaviors of the present G-QECCs with the perimeters $[[N=|V|, k, d]]$. For an arbitrary integer $m$, we can select the perimeter $k$ such that

$$
\eta|V|-1 \leq|V|-k \leq \eta|V|,
$$

where $0<\eta<1$. Therefore, it is easy to prove that

$$
\lim _{m,|V| \rightarrow \infty} \eta N=\infty ; \quad \lim _{m,|V| \rightarrow \infty} \inf \delta=\eta>0 ; \quad \lim _{m,|V| \rightarrow \infty} \inf R=1-\eta>0,
$$

which implies that the present graphical codes are asymptotically good by adjusted their parameters. ${ }^{21,22}$

According to the properties of the entropy, there is an real number $p=\eta_{0}(1 / 2<$ $\left.\eta_{0}<1\right)$ such that $\eta_{0}=H\left(G, \eta_{0}\right)$. Therefore, for any $\eta_{0}\left(1 / 2<\eta_{0}<1\right)$ we can always get

$$
1-H(G, \eta) \leq 1-\eta
$$

from which we gain

$$
\lim _{m,|V| \rightarrow \infty} \inf R=1-\eta \geq 1-H(G, \eta) .
$$

Associated with Eqs. (42) and (44), good graphical quantum codes should meet the asymptotic Gilbert-Varshamov bound. In addition, for any $\eta \in(1 / 2,1)$ we obtain

$$
1-\eta \leq 1-H(G, \eta / 2),
$$


which implies,

$$
\lim _{m,|V| \rightarrow \infty} \inf R=1-\eta \leq 1-H(G, \delta / 2) .
$$

In this case, good graphical quantum codes by properly selecting the entanglement relation matrices can meet asymptotic Hamming bound.

Furthermore, to obtain for meeting the bounds, another factor is the choice of subgraphs. Graph coloring problem is to find a coloring method with as few colors as possible. The minimum number of colors in a coloring of $G$ is the chromatic number $\mathcal{X}(G)$ of $G$. The chromatic entropy $H_{\mathcal{X}}(G, p)$ of the probabilistic graph $(G, p)$ is the minimum entropy of any of its colorings, which directly induces good graphical quantum codes.

\section{Conclusion}

Applying graphical approach provides a direct way to construct error correction codes. Because arbitrary graph does not always correspond to a graph state, in this paper, we generalize the method of constructing graphical quantum codes by entangling its subgraphs. Correspondingly, by applying the construction approach, the adjacent matrices of large graphical quantum nondegenerate codes, such as multi-vertex colorable graph and nested graph, can be easily obtained. Furthermore, because of its flexibility, the parameters can be adjusted according to the constrains of good graphical quantum codes. In summary, this approach presents a more general construction method for graphical quantum code.

\section{Acknowledgment}

This work is supported by the Natural Science Foundation of China (Nos. 60773085, 60801051), Key Subject Construction Project of Shanghai Dianji University (No. 10xkf01), and Shanghai Outstanding Youth Foundation (No. 11AZ12).

\section{References}

1. P. W. Shor, Phys. Rev. A. 52, 2493 (1995).

2. A. R. Calderbank and P. W. Shor, Phys. Rev. A 54, 1098 (1996).

3. A. M. Steane, Phys. Rev. Lett. 77, 793 (1996).

4. D. Gottesman, Phys. Rev. A 54, 1862 (1996).

5. M. Hein, J. Eisert and H. J. Briegel, quant-ph/0307130, (2004).

6. R. Raussendorf, D. E. Browne and H. J. Briegel, Phys. Rev. A 68, 022312 (2003).

7. H. J. Briegel and R. Raussendorf, Phys. Rev. Lett. 86, 910 (2001).

8. D. Schlingemann and R. F. Werner, Phys. Rev. A 65, 012308 (2002).

9. M. Hein, J. Eisert and H. J. Briegel, Phys. Rev. A 69, 062311 (2004).

10. S. Yu, Q. Chen and C. H. Oh, e-print arXiv:0709.1780.

11. L. E. Danielsen, quant-ph/0503236.

12. M. Van den Nest, J. Dehaene and B. De Moor, Phys. Rev. A 69, 022316 (2004).

13. D. Hu et al., Phys. Rev. A 78, 012306 (2008).

14. K. Chen, H. K. Lo, quant-ph/0404133. 
15. W. Dür, H. Aschauer and H. J. Briegel, Phys. Rev. Lett. 91, 107903 (2003).

16. H. Aschauer, W. Dür and H. J. Briegel, Phys. Rev. A 71, 012319 (2005).

17. C. Kruszynska et al., Phys. Rev. A 74, 052316 (2006).

18. Géza and O. Gühne, Phys. Rev. A 72, 022340 (2005).

19. R. C. Singleton, IEEE Trans. Inf. Theory IT-10, 116 (1964).

20. E. Hansler et al., Networks 4, (1974).

21. N. Ahmed and K. R. Rao, Orthogonal Transforms for Dignal Signal Processing (Springer-Verlag, Beilin, Germany, 1975).

22. R. Matsumoto, IEEE. Trans. Inf. Theory 48, 2122 (2002). 